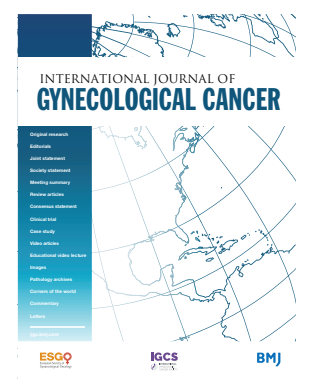

\title{
SUCCOR cone study: conization before radical hysterectomy
}

Enrique Chacon (D) , ${ }^{1}$ Nabil Manzour (1) , ${ }^{2}$ Vanna Zanagnolo, ${ }^{3}$ Denis Querleu (D),${ }^{4}$

Jorge M Núñez-Córdoba, ${ }^{5}$ Nerea Martin-Calvo, ${ }^{6}$ Mihai Emil Căpîlna, ${ }^{7}$ Anna Fagottii, ${ }^{8}$ Ali Kucukmetin, ${ }^{9}$ Constantijne Mom, ${ }^{10}$ Galina Chakalova, ${ }^{11}$ Aliyev Shamistan, ${ }^{12}$ Antonio Gil Moreno, ${ }^{13,14}$ Mario Malzoni, ${ }^{15}$ Fabrice Narducci, ${ }^{16}$ Octavio Arencibia, ${ }^{17}$ Francesco Raspagliesi, ${ }^{18}$ Tayfun Toptas (D),${ }^{19}$ David Cibula, ${ }^{20}$ Dilyara Kaidarova, ${ }^{21}$ Mehmet Mutlu Meydanli (D) ${ }^{22}$ Mariana Tavares, ${ }^{23}$ Dmytro Golub, ${ }^{24}$ Anna Myriam Perrone (D) , ${ }^{25}$ Robert Poka (D) , ${ }^{26}$ Dimitrios Tsolakidis, ${ }^{27}$ Goran Vujić, ${ }^{28}$ Marcin A Jedryka (D), ${ }^{29}$ Petra L M Zusterzeel, ${ }^{30}$ Jogchum Jan Beltman, ${ }^{31}$ Frederic Goffin, ${ }^{32}$ Dimitrios Haidopoulos, ${ }^{33}$ Herman Haller, ${ }^{34}$ Robert Jach, ${ }^{35}$ Iryna Yezhova, ${ }^{36}$ Igor Berlev, ${ }^{37}$ Margarida Bernardino, ${ }^{38}$ Rasiah Bharathan, ${ }^{39}$ Maximilian Lanner, ${ }^{40}$ Minna M Maenpaa, ${ }^{41}$ Vladyslav Sukhin (D) ${ }^{42}$ Jean-Guillaume Feron, ${ }^{43}$ Robert Fruscio (D) ,44,45 Kersti Kukk, ${ }^{46}$ Jordi Ponce, ${ }^{47}$ Jose Angel Minguez (D), ${ }^{48}$ Daniel Vázquez-Vicente (D) ${ }^{49}$ Teresa Castellanos, ${ }^{49}$ Felix Boria (D) , Juan Luis Alcazar (D), ${ }^{51}$ Luis Chiva (D),${ }^{52}$ The SuccoR study group

For numbered affiliations see end of article.

Correspondence to Dr Luis Chiva, Obstetrics and Gynecology, Clinica Universidad de Navarra, MADRID, Spain; Ichiva@unav.es

Received 28 February 2021 Accepted 6 December 2021 Published Online First 17 January 2022

\section{SLinked}

- http://dx.doi.org/10.1136/ ijgc-2021-003315

Check for updates

(C) IGCS and ESGO 2022. No commercial re-use. See rights and permissions. Published by BMJ.

To cite: Chacon $\mathrm{E}$, Manzour N, Zanagnolo V, et al. Int J

Gynecol Cancer 2022;32:117124.

\section{HIGHLIGHTS}

- Patients with prior cervical conization had a significantly lower risk of relapse and death

- Patients who underwent minimally invasive surgery without conization had a higher chance of relapse

- Patients with prior conization and minimally invasive surgery had similar outcomes compared with the open approach

\section{ABSTRACT}

Objective To evaluate disease-free survival of cervical conization prior to radical hysterectomy in patients with stage IB1 cervical cancer (International Federation of Gynecology and Obstetrics (FIG0) 2009).

Methods A multicenter retrospective observational cohort study was conducted including patients from the Surgery in Cervical Cancer Comparing Different Surgical Aproaches in Stage IB1 Cervical Cancer (SUCCOR) database with FIGO 2009 IB1 cervical carcinoma treated with radical hysterectomy between January 1, 2013, and December 31, 2014. We used propensity score matching to minimize the potential allocation biases arising from the retrospective design. Patients who underwent conization but were similar for other measured characteristics were matched 1:1 to patients from the non-cone group using a caliper width $\leq 0.2$ standard deviations of the logit odds of the estimated propensity score.

Results We obtained a weighted cohort of 374 patients (187 patients with prior conization and 187 nonconization patients). We found a $65 \%$ reduction in the risk of relapse for patients who had cervical conization prior to radical hysterectomy (hazard ratio (HR) 0.35 , $95 \%$ confidence interval (Cl) 0.16 to $0.75, p=0.007$ ) and a $75 \%$ reduction in the risk of death for the same sample (HR $0.25,95 \% \mathrm{Cl} 0.07$ to $0.90, \mathrm{p}=0.033$ ). In addition, patients who underwent minimally invasive surgery without prior conization had a 5.63 times higher chance of relapse compared with those who had an open approach and previous conization (HR 5.63, 95\% Cl 1.64 to $19.3, p=0.006)$. Patients who underwent minimally invasive surgery with prior conization and those who underwent open surgery without prior conization showed no differences in relapse rates compared with those who underwent open surgery with prior cone biopsy (reference)
(HR 1.94, 95\% Cl 0.49 to $7.76, p=0.349$ and $H R 2.94,95 \%$ $\mathrm{Cl} 0.80$ to $10.86, p=0.106$ respectively).

Conclusions In this retrospective study, patients undergoing cervical conization before radical hysterectomy had a significantly lower risk of relapse and death.

\section{INTRODUCTION}

For decades, the main indication for cervical conization has been the treatment of pre-invasive cervical disease. This technique has also been shown to be a useful diagnostic tool in cases of microinvasive disease, usually having no role in patients with macroscopic tumors. Furthermore, in selected patients with early cervical cancer, cone biopsy has been indicated as a fertility sparing therapeutic approach with curative intent. ${ }^{1}$

Recently, after the publication of the Laparoscopic Approach to Cervical Cancer (LACC) trial, ${ }^{2}$ there has been a paradigm shift in the surgical approach of early cervical cancer towards the open approach. In this scenario, Chiva et al published the Surgery in Cervical Cancer Comparing Different Surgical Aproaches in Stage IB1 Cervical Cancer (SUCCOR) study ${ }^{3}$; they concluded that minimally invasive surgery for cervical cancer increased the risk of relapse and death compared with open surgery. In this retrospective study, women who had undergone conization before surgery were excluded from the analysis as a possible source of bias. To the best of our knowledge, neither the LACC trial or other retrospective studies ${ }^{4-10}$ have evaluated the potential confounding effect of conization before surgery. 


\section{Original research}

On the other hand, three recent studies ${ }^{11-13}$ have found that cervical conization may be a significant independent predictor of the risk of relapse. Therefore, the primary objective of this study was to establish the potential impact of conization before radical hysterectomy on disease-free survival in patients with early cervical cancer. Second, we aimed to analyze the effect of conization on the overall survival of this group of patients. Finally, we compared the risk of relapse according to the surgical approach and conization status.

\section{METHODS}

\section{Accrual and Data Source}

We used information from the SUCCOR database ${ }^{3}$ to complete these objectives. From May 15, 2019, to November 15, 2019, we collected data from 1272 patients with stage IB1 cervical cancer (International Federation of Gynecology and Obstetrics (FIG0) 2009) ${ }^{14}$ who underwent a radical hysterectomy in Europe from January 1, 2013, to December 31, 2014. Researchers from 126 institutions in 29 European countries registered and contributed to the project. After obtaining ethical consent from our central institutional review board, we required a certificate of approval or a letter of exemption from the local ethics committees from all the investigators.

\section{Inclusion and Exclusion Criteria}

The inclusion criteria were ${ }^{15}$ : age $\geq 18$ years; histologic type: squamous cell carcinoma, adenocarcinoma, or adenosquamous carcinoma; and pelvic magnetic resonance image (MRI) confirming a tumor diameter $\leq 4 \mathrm{~cm}$ with no parametrial invasion and a preoperative computerised tomography (CT) scan, MRI, or positron emission tomography-computed tomography (PET-CT) demonstrating no extracervical metastatic disease. The operative report had to describe type B-C radical hysterectomy with bilateral pelvic lymphadenectomy by either minimally invasive surgery (laparoscopic or robotic) or open surgery, including at least 10 pelvic nodes. Women who underwent sentinel lymph node mapping plus pelvic lymphadenectomy were also included in the study.

Patients were excluded if any other histological type was diagnosed, if tumor size was $>4 \mathrm{~cm}$ in the pathology report, or if there was a past history of any invasive tumor, previous chemotherapy or radiation, suspicious lymph nodes, or metastatic disease on preoperative imaging. Conversion from minimally invasive to laparotomy was cause for exclusion (following the exclusion criteria established in the original SUCCOR study), as well as if the patient was pregnant at diagnosis.

\section{Outcomes}

Disease-free survival was defined as the time from the date of radical hysterectomy to the time of relapse or last contact, whichever came first. The relapse was diagnosed by physical examination as well as by imaging and biopsy (in 23 of 34 cases $(67.7 \%$ ) relapse was demonstrated by histological confirmation). Overall survival was calculated from the radical hysterectomy date to the time of the last contact or death from cervical cancer, whichever came first.

\section{Statistical Analysis}

We used propensity score matching to minimize the potential allocation biases arising from the study's retrospective design. We ran a multivariable logistic regression model using conization as a dependent variable to identify the potential confounders that would be considered to calculate the propensity score. The following variables were included in the model: surgical approach, largest tumor diameter in the pathological specimen, tumor grade, lymphovascular invasion, depth of invasion, margin status, nodal status, and adjuvant therapy. Patients who underwent conization but were similar concerning other measured characteristics were matched 1:1 to patients from the no-cone biopsy group using a caliper width $\leq 0.2$ standard deviations (SDs) of the logit odds of the estimated propensity score.

Basic descriptive statistics were used to describe the sample. The follow-up time was summarized using the median, 25th percentile (p25), and 75th percentile (p75). Differences in categorical variables were analyzed using the $\chi^{2}$ test. Continuous variables were compared using the Student's t test. Survival outcomes (disease-free survival and overall survival) were estimated using the Kaplan-Meier model. The log rank test was used to compare the risk of recurrence and the risk of death between groups. We used weighted Cox proportional hazards to calculate the hazard ratio (HR) and 95\% confidence interval (Cl) for disease-free survival and overall survival using as a reference the group of women who did not undergo a cone biopsy (except for Figure 4, where the reference group was patients who were operated on by an open approach with prior cone biopsy).

The hazard's proportionality was assessed based on Schoenfeld residuals $(p=0.834)$. All analyses were performed with the IBM SPSS 26.0 and the Stata 14 packages. All p values are two sided. Statistical significance was defined a priori as $p<0.05$.

\section{RESULTS}

\section{Entire Cohort Characteristics}

A total of 116 patients did not meet the inclusion criteria or had missing information on follow-up and were excluded from the study. We performed subsequent analyses in 1156 patients. Of these patients, $423(36.6 \%)$ underwent prior conization before radical hysterectomy and $733(63.4 \%)$ underwent radical hysterectomy without previous cervical conization.

We performed a propensity matched comparison (1:1) comparing 187 patients with cone biopsy prior to radical hysterectomy with 187 without prior to cervical conization. Figure 1 details the study design. Table 1 shows the patient characteristics in both groups. Median follow-up time was 59 months (p25-p75 47-66) for nonconization and 58 months (48-65) for conization. Baseline characteristics were similar between groups as a result of a propensity matched comparison (Table 1).

Mean age in the non-conization group was 48.1 years (SD 9.59) versus 45.8 (SD 10.22) in the conization group. Mean body mass index was $26.44 \mathrm{~kg} / \mathrm{m}^{2}$ (SD 6.18) and 25.05 (SD 4.64), respectively. Regarding the surgical approach, a total of 97 (51.9\%) radical hysterectomies were performed by laparotomy and 90 (48.1\%) by minimally invasive surgery in the non-conization group versus 94 (50.3\%) and 93 (49.7\%), respectively, in the conization group. Among patients who underwent minimally invasive surgery, $18.7 \%$ (17 patients) were operated on robotically in the conization group compared with $6.4 \%$ (6 patients) in the non-conization group. 


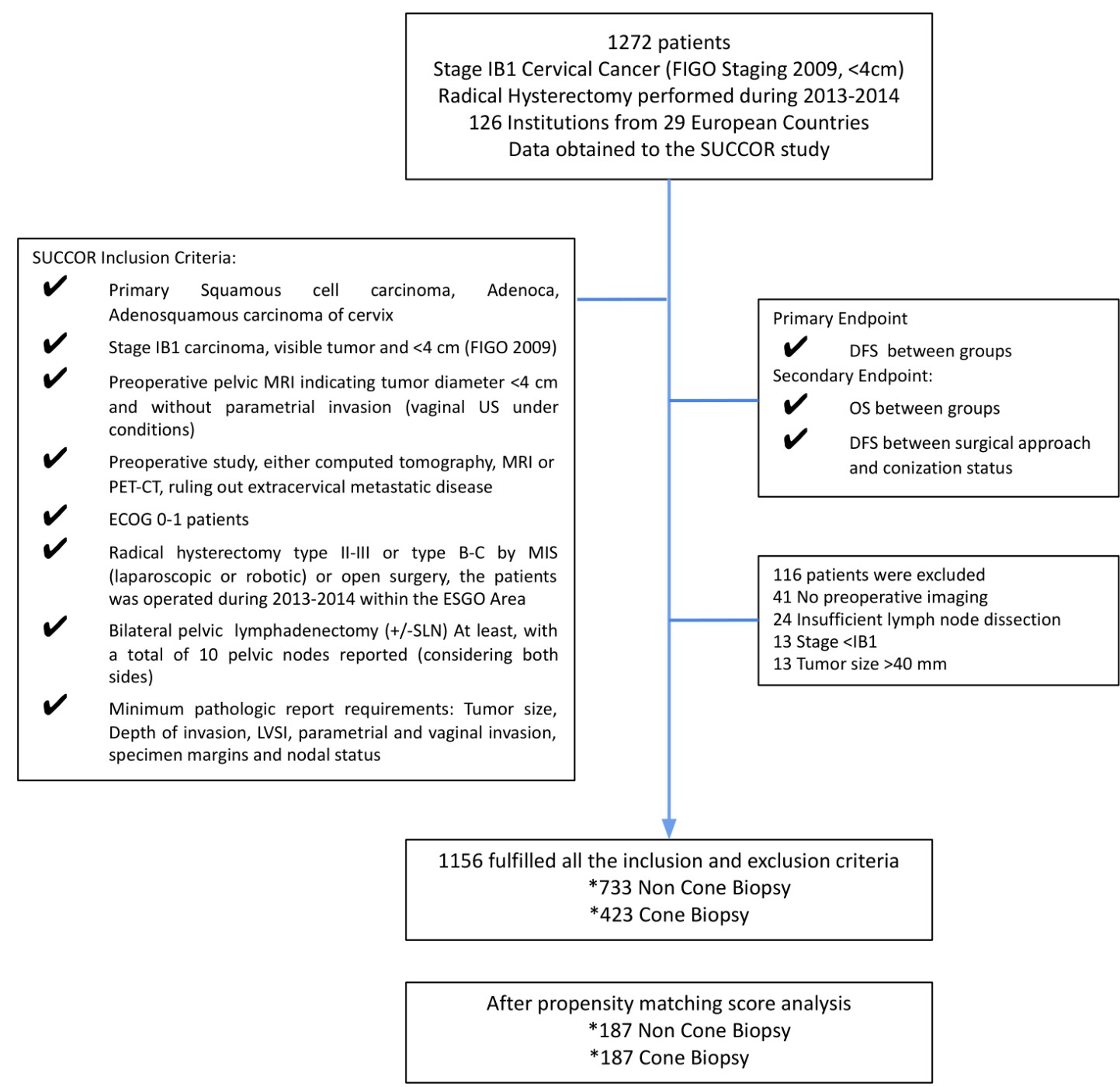

Figure 1 Flowchart of study population. DFS, disease-free survival; ECOG, Eastern Cooperative Oncology Group; ESGO, European Society of Gynaecological Oncology; FIGO, International Federation of Gynecology and Obstetrics; LVSI, lymphovascular space invasion; MRI, magnetic resonance imaging; OS, overall survival; PET-CT, positron emission tomography-computed tomography; SLN, sentinel lymph node; US, ultrasound.

A senior surgeon with more than 10 years' experience was the first surgeon in $150(80.2 \%)$ and $148(70.1 \%)$ procedures in both groups. In most (67.7\% and $71.5 \%$, respectively) procedures, no manipulator was used. Colpoprotective maneuvers were only performed in $18.7 \%$ of the procedures in both groups.

The most common histologic tumor type was squamous carcinoma (131 $(70.1 \%)$ in both groups) and most were smaller than $2 \mathrm{~cm}(119(63.6 \%)$ vs $133(71.1 \%)$, respectively). Median tumor size in the non-conization group was $19 \mathrm{~mm}$ (SD 9.45) vs 12 (10.94) in the conization group. Lymphovascular space invasion was present in 73 patients $(39.0 \%)$ in the non-conization group and in 77 patients $(41.2 \%)$ in the prior conization group. Deep stromal invasion was diagnosed in $20.3 \%$ of cases in both groups ( 38 cases per group). Parametrial invasion was observed only in $1.5 \%$ and $0.5 \%$ of patients, respectively. A total of 14 patients $(7.5 \%)$ had nodal metastasis in the non-conization group compared with $18(9.6 \%)$ in the conization group.

Eighty (42.8\%) patients received adjuvant therapy in the nonconization group compared with $79(42.2 \%)$ in the conization group. Standard external radiation and brachytherapy were the most frequently used modalities of adjuvant treatment $(47.5 \%$ and $45 \%$ in the non-conization group vs $58.2 \%$ and $58.2 \%$ in the conization group), while concomitant chemoradiation was used in $38.7 \%$ and $32.9 \%$, respectively.

\section{Conization Group}

Table 2 shows the patient characteristics in the conization cohort. Regarding the technique used, the majority (135 patients (72.2\%)) was performed by the loop electrosurgical excision procedure (LEEP)/large loop excision of the transformation zone (LLETZ), with a lower proportion performed by cold knife or laser $(33$ (17.5\%) and $10(5.3 \%)$ patients, respectively). Median time from conization to radical hysterectomy was 50.1 days (range 1-138).

Of the 187 patients who underwent conization, $26(13.9 \%)$ and $145(77.5 \%)$ had negative and positive surgical margins on the cone biopsy specimen, respectively. In addition, 151 (80.7\%) patients who underwent conizations had residual disease in the final hysterectomy specimen. Despite this, there was no difference in disease 


\section{Original research}

\begin{tabular}{|c|c|c|c|}
\hline Baseline characteristics & Non-conization $(n=187)$ & Conization $(n=187)$ & $P$ value \\
\hline Age (years) (mean (SD)) & $48.11(9.59)$ & $45.85(10.22)$ & 0.91 \\
\hline Body mass index $\left(\mathrm{kg} / \mathrm{m}^{2}\right)($ mean $(\mathrm{SD}))$ & $26.44(6.18)$ & $25.05(4.64)$ & 0.12 \\
\hline Race $(n(\%))$ & & & 0.36 \\
\hline Caucasian & $155(82.9)$ & $165(88.2)$ & \\
\hline Asian & $6(3.2)$ & $8(4.3)$ & \\
\hline Latin American & $5(2.7)$ & $2(1.1)$ & \\
\hline African & $2(1.1)$ & $0(0.0)$ & \\
\hline Not reported & $19(10.1)$ & $12(6.4)$ & \\
\hline Performance status (n (\%)) & & & 0.12 \\
\hline ECOG 0 & $166(88.8)$ & $174(93.0)$ & \\
\hline ECOG 1 & $16(8.6)$ & $6(3.2)$ & \\
\hline Not reported & $5(2.6)$ & $7(3.8)$ & \\
\hline Approach (n (\%)) & & & 0.76 \\
\hline MIS & $90(48.1)$ & $93(49.7)$ & \\
\hline Robotic & $6(6.4)$ & $17(18.7)$ & \\
\hline Open & $97(51.9)$ & $94(50.3)$ & \\
\hline Colpoprotective maneuvers ( $(\%))$ & $35(18.7)$ & $35(18.7)$ & 1 \\
\hline No use of uterine manipulator ( $\mathrm{n}(\%))$ & $109(67.7)$ & $123(71.5)$ & 0.45 \\
\hline Histotype (n (\%)) & & & 0.83 \\
\hline Squamous & $131(70.1)$ & $131(70.1)$ & \\
\hline Adenocarcinoma & $49(26.2)$ & $51(27.3)$ & \\
\hline Adenosquamous & $7(3.7)$ & $5(2.7)$ & \\
\hline Tumor grade (n (\%)) & & & 0.34 \\
\hline G1 & $35(18.7)$ & $42(22.5)$ & \\
\hline G2 & $96(51.3)$ & $82(43.9)$ & \\
\hline G3 & $56(29.9)$ & $63(33.7)$ & \\
\hline Largest tumor diameter (n (\%)) & & & 0.12 \\
\hline$\leq 2 \mathrm{~cm}$ & $119(63.6)$ & $133(71.1)$ & \\
\hline$>2 \mathrm{~cm}$ & $68(36.4)$ & $54(28.9)$ & \\
\hline Depth of invasion (n (\%)) & & & 0.78 \\
\hline Superficial $<1 / 3$ & 69 (36.9) & 75 (40.1) & \\
\hline Intermediate $1 / 3-2 / 3$ & $80(42.8)$ & 74 (39.6) & \\
\hline Deep $>2 / 3$ & $38(20.3)$ & $38(20.3)$ & \\
\hline Lymphovascular space invasion (n (\%)) & & & 0.67 \\
\hline No & $114(61.0)$ & $110(58.8)$ & \\
\hline Yes & $73(39.0)$ & $77(41.2)$ & \\
\hline Parametrial invasion (n (\%)) & & & 0.32 \\
\hline Negative & $183(98.0)$ & $182(97.3)$ & \\
\hline Positive & $3(1.5)$ & $1(0.5)$ & \\
\hline Not reported & $1(0.5)$ & $4(2.2)$ & \\
\hline Margins status of radical hysterectomy specimen ( $\mathrm{n}(\%))$ & & & 1 \\
\hline Negative & $175(93.6)$ & $175(93.6)$ & \\
\hline Positive or close $\leq 2 \mathrm{~mm}$ & $12(6.4)$ & $12(6.4)$ & \\
\hline Nodal status (n (\%)) & & & 0.46 \\
\hline Negative & $173(92.5)$ & $165(90.4)$ & \\
\hline Positive & $14(7.5)$ & $18(9.6)$ & \\
\hline
\end{tabular}


Table 1 Continued

\begin{tabular}{lcc}
\hline Baseline characteristics & Non-conization $(\mathbf{n = 1 8 7})$ & Conization $(\mathbf{n}=\mathbf{1 8 7})$ \\
\hline FIGO staging $2009(\mathrm{n}(\%))$ & & $94(50.3)$ \\
IB1 $\leq 2 \mathrm{~cm}$ & $103(55.1)$ & $75(40.1)$ \\
IB1 $>2 \mathrm{~cm}$ & $65(34.8)$ & $18(9.6)$ \\
IB2-II-III & $19(10.2)$ & \\
Adjuvant therapy (n (\%)) & $108(57.8)$ \\
$\quad$ None & $107(57.2)$ & $79(42.2)$ \\
Yes & $80(42.8)$ & 0.91 \\
\hline
\end{tabular}

ECOG, Eastern Cooperative Oncology Group; FIGO, International Federation of Gynecology and Obstetrics; MIS, minimally invasive surgery.

recurrence between patients with positive or negative conization margins $(92.3 \%$ and $95.1 \%$, respectively, $\log$ rank $p=0.78)$.

\section{Oncologic Outcomes}

There were 9 (4.9\%) relapses in 183 patients in the cone biopsy group. In the group without conization, there were $25(14 \%)$ relapses in 178 patients $(p=0.003)$. After a median follow-up of 58 months (p25-p75 48-66), disease-free survival was 95.1\% in the conization group and $86 \%$ in the non-conization group. A $65 \%$ reduction in the risk of relapse was observed for patients who had undergone a cervical conization ( $\mathrm{HR} 0.35,95 \% \mathrm{Cl} 0.16$ to 0.75 , $\mathrm{p}=0.005$ ) (Figure 2).

Table 2 Specific characteristics in the conization group

\begin{tabular}{lc}
\hline Baseline characteristics ( $\mathbf{n = 1 8 7})$ & $\begin{array}{l}\text { Conization } \\
\text { group }\end{array}$ \\
\hline $\begin{array}{l}\text { Time from conization to radical } \\
\text { hysterectomy (days) (median (range)) }\end{array}$ & $50.1(1-138)$ \\
Type of cone (n (\%)) & \\
$\quad$ LEEP/LLETZ & $135(72.2)$ \\
Cold knife & $33(17.5)$ \\
Laser & $10(5.3)$ \\
$\quad$ Not reported & $9(5.0)$ \\
Residual tumor in final radical & \\
hysterectomy specimen (n (\%)) & \\
$\quad$ None & $36(19.3)$ \\
Yes & $151(80.7)$ \\
Margins status of conization specimen & \\
(n (\%)) & \\
Uncertain & $6(3.2)$ \\
Negative & $26(13.9)$ \\
Positive & $145(77.5)$ \\
Not reported & $10(5.4)$ \\
Disease-free survival by cone margins & Log rank p=0.783 \\
status ( $(\%)$ ) & \\
Negative & 92.3 \\
Positive & 95.1 \\
\hline
\end{tabular}

LEEP, loop electrosurgical excision procedure; LLETZ, large loop excision of the transformation zone.
Patients who underwent radical hysterectomy without prior conization had a lower overall survival rate than patients with cone biopsy. A total of $3(1.6 \%)$ patients died in the conization group while $12(6.6 \%)$ patients died in the non-conization group $(\mathrm{p}=0.015)$. After a median follow-up of 59 months (p25-p75, 50-66), overall survival was $93.4 \%$ in the non-conization group and $98.4 \%$ in patients who had prior conization. A $75 \%$ reduction in the risk of death was seen for patients who had a previous cone biopsy (HR $0.25,95 \% \mathrm{Cl} 0.07$ to $0.90, \mathrm{p}=0.021$ ) (Figure 3).

Finally, patients who underwent minimally invasive surgery without prior conization had a 5.63 times higher chance of relapse compared with those who underwent an open approach with previous conization (HR 5.63, 95\% Cl 1.64 to $19.3, p=0.006$ ) (Figure 4).

Patients who underwent minimally invasive surgery with prior conization and those who underwent open surgery without prior conization showed no differences in relapse rates compared with those who underwent open surgery with prior cone biopsy (reference) (HR 1.94, 95\% Cl 0.49 to $7.76, p=0.35$ and $\mathrm{HR} 2.94,95 \% \mathrm{Cl}$ 0.80 to $10.86, p=0.11$, respectively).

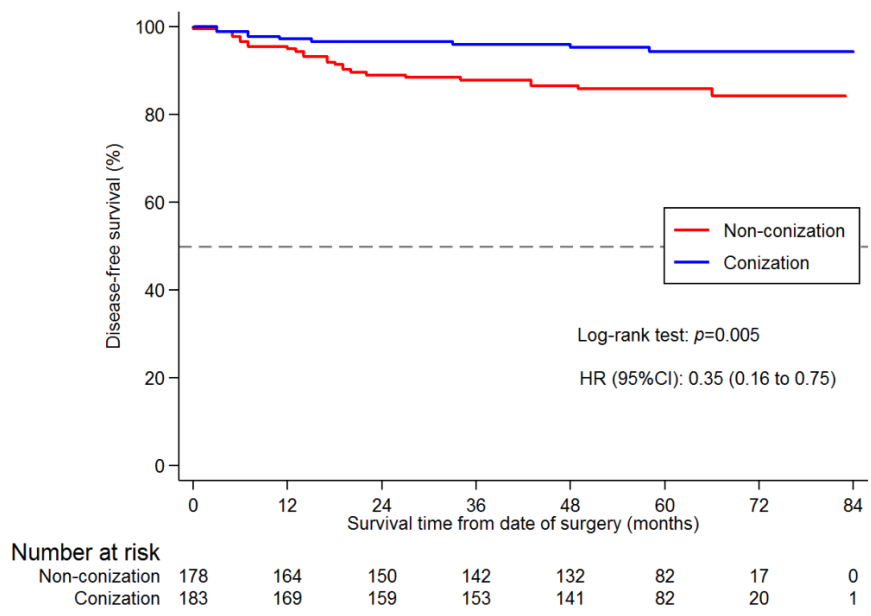

Figure 2 Hazard ratios (HR) and 95\% confidence intervals for the risk of relapse by type of conization (with or without). 


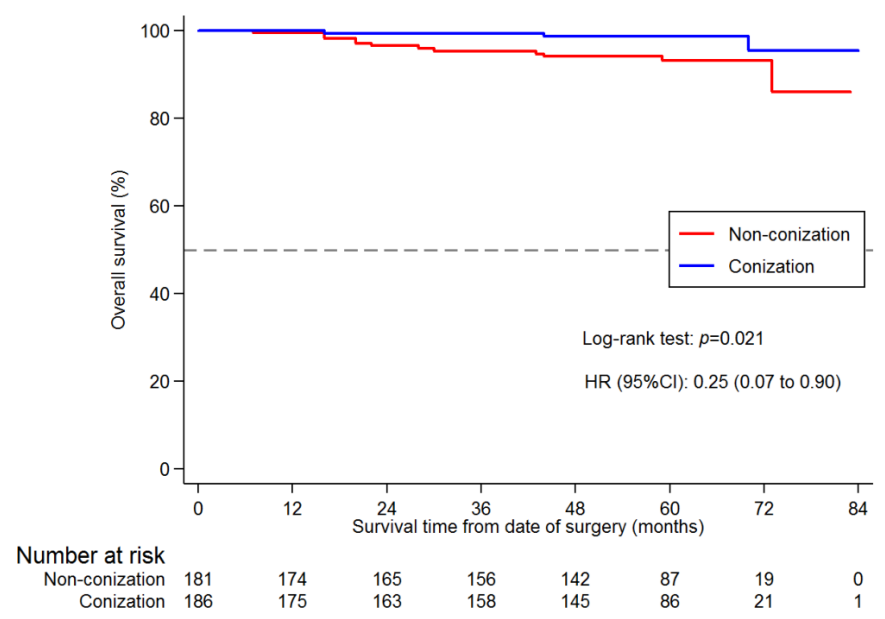

Figure 3 Hazard ratios (HR) and 95\% confidence intervals for overall survival by type of conization (with or without).

\section{DISCUSSION}

\section{Summary of Main Results}

In this retrospective study, we showed that patients with stage IB1 cervical cancer (FIGO 2009) who underwent prior cervical conization followed by radical hysterectomy had a significantly lower risk of relapse ( $\mathrm{HR} 0.35,95 \% \mathrm{Cl} 0.16$ to $0.75, \mathrm{p}=0.005$ ) and death (HR $0.25,95 \% \mathrm{Cl} 0.07$ to $0.90, \mathrm{p}=0.021$ ). In addition, those patients who underwent minimally invasive surgery without prior conization had a 5.63 times higher chance of relapse compared with those who underwent an open approach and previous conization (HR 5.63, 95\% Cl 1.64 to 19.3, $p=0.006$ ). Patients who underwent minimally invasive surgery with prior conization had no differences in relapse rates compared with those who underwent open surgery with prior cone biopsy (HR 1.94, 95\% Cl 0.49 to $7.76, p=0.349$ ).

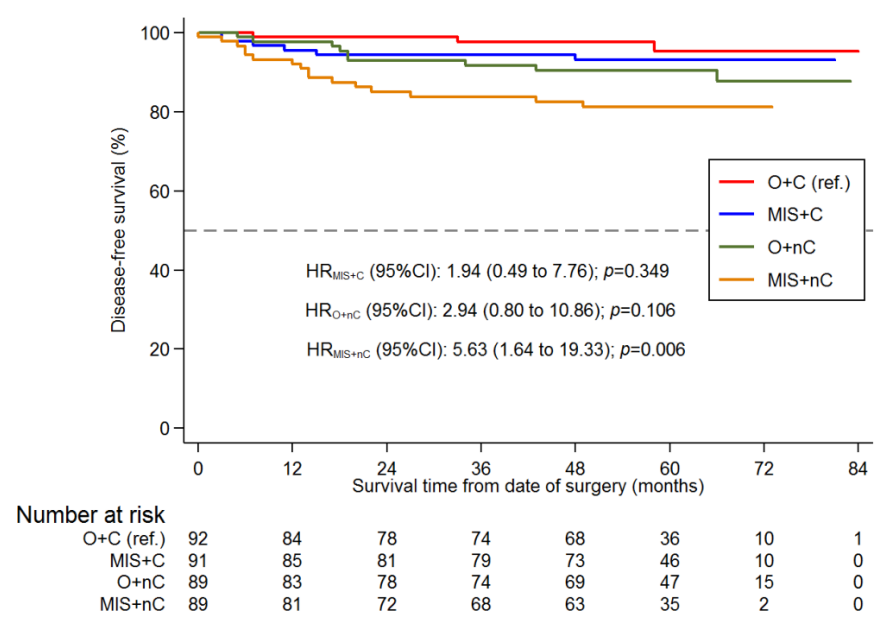

Figure 4 Hazard ratios (HR) and 95\% confidence intervals for the risk of relapse according to surgical approach and conization status. MIS $+\mathrm{C}$, patients who underwent minimally invasive surgery with prior conization; MIS $+n C$, patients who underwent minimally invasive surgery without prior conization; $\mathrm{O}+\mathrm{C}$, patients who underwent open surgery with prior conization; $\mathrm{O}+\mathrm{nC}$, patients who underwent open surgery without prior conization.

\section{Results in the Context of Published Literature}

Few studies have analyzed the prognostic role of conization before radical hysterectomy; in particular, three recent studies demonstrated that conization was associated with reduced recurrence rates. ${ }^{11-13}$ Uppal et al $^{11}$ analyzed 243 patients who underwent conization and had no residual tumor on preoperative imaging; there was no difference in relapse rate between the open $(n=72)$ and minimally invasive surgery $(n=171)$ groups $(1.4 \%$ vs $2.9 \%, p=0.48)$.

Casarin et $a^{12}$ identified 186 patients with FIGO 2009 stage IA1-IB1 disease who underwent minimally invasive radical hysterectomy and reported that performance of preoperative conization was associated with a lower risk of relapse $(1.1 \%$ vs $16.1 \%$, $p<0.001)$, even for patients with stage IB1 disease $(1.8 \%$ vs $17.2 \%, p=0.004)$. Another study, conducted by Bizarri et al, ${ }^{16}$ noted the possible protective role of conization in early stage cervical cancer patients after propensity score matching. The investigators observed that patients with prior conization received less adjuvant treatment $(p<0.001)$ and had better disease-free survival than patients who did not undergo conization $(89.8 \%$ vs $80.0 \%$, respectively; $p=0.010)$. No differences in 5 -year overall survival $(97.1 \%$ vs $91.4 \%$, respectively; $p=0.114$ ) and the recurrence pattern $(p=0.115)$ were reported between the two groups.

Finally, a recently published prospective trial (ConCerv trial) ${ }^{17}$ aimed to assess the feasibility of conservative surgery in women with low risk cervical cancer (tumor size $<2 \mathrm{~cm}$, no lymphovascular space invasion, depth of invasion $<10 \mathrm{~mm}$, and negative pelvic nodes). In a secondary analysis of the study, Schmeler et al observed that the recurrence rate in patients undergoing inadvertent simple hysterectomy without prior conization (with unexpected postoperative diagnosis of invasive cancer) plus subsequent lymph node dissection (16 patients) was $12.5 \%$ versus $0 \%$ in those with a known preoperative diagnosis of invasive cancer who underwent simple hysterectomy preceded by prior conization (40 patients).

\section{Strengths and Weaknesses}

Overall, the strengths of this study include a collaborative effort of 126 European institutions from 29 countries where comprehensive data were collected on 1272 patients. Another strength of our study was the use of propensity score matching in an attempt to balance confounders between groups, obtaining a weighted population, such that selection bias for the surgical procedure was minimized. It is also important to emphasize that our study is, to the best of our knowledge, the first study showing that patients with prior conization followed by radical hysterectomy had a significantly lower risk of relapse and death. However, we recognize that such groups, by definition, may already be at a lower risk of relapse.

Our study has several weaknesses due to the retrospective nature, including the fact that there was no formal auditing of the data. To account for these limitations, we provided the participating sites with a strict list of inclusion and exclusion criteria, ${ }^{315}$ and all investigators declared that the reported information adhered to the data in the reviewed charts. Moreover, there was no information regarding indications for conization and surgical approach. Also, it should be mentioned that cervical conization was excluded from the original SUCCOR study ${ }^{3}$ for the following reasons: cone biopsies were often performed at outside institutions, leading to missing critical pathological information; insufficient pathology report to stage the tumor adequately; lack of consensus on measurement 
of tumor size when the cone biopsy and final specimen both had residual disease; and if no residual tumor, then risk for tumor dissemination may be lower than that of patients with gross tumor. For these reasons, the inclusion of this group of patients may have represented a possible source of bias.

Similarly, our study did not provide differential evaluation of patients with stage IB1 disease based on microscopic disease versus gross tumor on cervical specimen. Lastly, there were no data on the regimen used for surveillance or information as to whether recurrences were documented by clinical suspicion, imaging studies, or pathologic confirmation. This, together with the small number of events, represents a further limitation.

\section{Implications for Practice and Future Research}

Considering the potential benefit in terms of relapse and survival of patients undergoing conization together with the diagnostic information that may enhance our ability to make the appropriate recommendation, ${ }^{18}{ }^{19}$ we believe that cervical conization, especially in small tumors (up to $2 \mathrm{~cm}$, conization has no role in large tumors), may be used for tailoring the surgery and the choice of surgical approach in cervical cancer patients.

Regarding the potential protective effect of conization, after the publication of the LACC trial, ${ }^{2}$ the reasons for minimally invasive surgery having worse oncological outcomes have not been well established. In this context and taking into consideration that it was a secondary objective of our studies, the analyses of the SUCCOR group may suggest the hypothesis that in minimally invasive surgery, the lesser exposure and manipulation of the tumor, whether by prior diagnostic conization (in our balanced population when a previous conization was performed, no differences were observed between the minimally invasive and open routes (HR $1.94,95 \% \mathrm{Cl} 0.49$ to $7.76, \mathrm{p}=0.349$ )) or by abandoning the manipulator $^{3}$ and implementing protective maneuvers such as vaginal closure, ${ }^{320}$ help to reduce tumor spillage.

For all of these reasons, the present study should be regarded as hypothesis generating to stimulate an international collaboration to investigate prospectively the potential role of conization, validating the results obtained by our group. Meanwhile, we believe that from now on, in all prospective and retrospective studies that are carried out, cervical conization should be taken into account as a variable that should be weighed for the risk of relapse.

\section{CONCLUSIONS}

In this retrospective study, cervical conization before radical hysterectomy was associated with improved disease-free survival and overall survival in patients with IB1 cervical cancer (FIG0 2009) treated with primary radical surgery, compared with patients who did not undergo conization.

\footnotetext{
Author affiliations

${ }^{1}$ Gynecologic Oncology, Universidad de Navarra, Pamplona, Navarra, Spain

${ }^{2}$ Clinica Universidad de Navarra Departamento de Ginecologia y Obstetricia, Pamplona, Navarra, Spain

${ }^{3}$ Istituto Europeo di Oncologia, Milano, Anognnn, Italy

${ }^{4}$ Surgery, Institut Bergonie, Bordeaux, France

${ }^{5}$ Clinica Universidad de Navarra, Pamplona, Navarra, Spain

${ }^{6}$ Department of Preventive Medicine and Public Health, Universidad de Navarra,

Pamplona, Spain
}

${ }^{7}$ Emergency County Hospital Targu-Mures, Targu Mures, Romania

${ }^{8}$ University Hospital Agostino Gemelli Gynaecological Oncology Complex Operative Unit, Roma, Lazio, Italy

${ }^{9}$ Queen Elizabeth Hospital, Gateshead, Gateshead, UK

${ }^{10}$ Amsterdam University Medical Centres, Amsterdam, Noord-Holland, Netherlands

${ }^{11}$ University Oncologic Hospital, Sofia, Bulgaria

${ }^{12}$ National Center of Oncology, Baku, Azerbaijan

${ }^{13}$ Gynecology, Vall d'Hebron Hospital, Sant Cugat Del Vallés, Barcelona, Spain

${ }^{14}$ Universitat Autònoma de Barcelona, Barcelona, Barcelona, Spain

${ }^{15}$ Endoscopica Malzoni, Center for Advanced Endoscopic Gynecologic Surgery, Center for Advanced Endoscopic Gynecologic Surgery, Avellino, Italy

${ }^{16}$ Department of Gynecology, Centre Oscar Lambret, Lille, France

${ }^{17}$ University Maternal Hospital Canary Islands, Las Palma, Spain

${ }^{18}$ Surgery, Fondazione IRCCS Istituto Nazionale dei Tumori, Milano, Lombardia, Italy

${ }^{19}$ Gynecologic Oncology, Saglik Bilimleri University Antalya Research and Training Hospital, Antalya, Turkey

${ }^{20}$ Department of Obstetrics and Gynecology, University of Prague, Prague, Czech

Republic

${ }^{21}$ Oncogynecology, Kazahskij Naucno-issledovatel'skij Institut Onkologii i Radiologii, Almaty, Kazakhstan

${ }^{22}$ Department of Gynecologic Oncology, Zekai Tahir Burak Women's Health

Education and Research Hospital, Ankara, Turkey

${ }^{23}$ IPO-PORTO, Porto, Porto, Portugal

${ }^{24}$ Department of Surgery, LISOD-Israeli Oncological Hospital, Kyiv, Ukraine

${ }^{25}$ Azienda Ospedaliero-Universitaria di Bologna Policlinico SantOrsola-Malpighi,

Bologna, Italy

${ }^{26}$ Obstetrics and Gynecology, Unit of Gynecologic Oncology, Institute of Obstetrics and Gynecology, Faculty of Medicine, University of Debrecen, Debrecen, Hungary

${ }^{27}$ General Hospital of Thessaloniki Papageorgiou, Thessaloniki, Central Macedonia, Greece

${ }^{28}$ Clinical Hospital Center, Zagreb, Croatia

${ }^{29}$ Oncological Gynecology, Lower Silesian Cancer Center, Wroclaw, Poland

${ }^{30}$ Gynecological Oncology, Radboudumc, Nijmegen, Netherlands

${ }^{31}$ Gynaecology, LUMC, Leiden, Netherlands

${ }^{32}$ Obstetrics and Gynecology, University of Liege, Liege, Belgium

${ }^{33}$ Division of Gynecologic Oncology, 1st Department of Obstetrics and Gynecology, Alexandra Hospital, National and Kapodistrian University of Athens, Athens, Greece

${ }^{34}$ Clinical Hospital Center Rijeka, Rijeka, Croatia

${ }^{35}$ Department of Gynecology and Oncology, Jagiellonian University, Krakow, Poland

${ }^{36}$ Lviv Oncology Center, Lviv, Ukraine

${ }^{37}$ North-Western State Medical University, NN Petrov Research Institute of

Oncology, Saint-Petersburg, Saint-Petersburg, Russian Federation

${ }^{38}$ Gynecology, Instituto Português de Oncologia de Lisboa, Lisboa, Lisboa, Portugal

${ }^{39}$ University Hospitals of Leicester NHS Trust, Leicester, UK

${ }^{40}$ Kardinal Schwarzenberg'sches Krankenhaus, Schwarzach, Steiermark, Austria

${ }^{41}$ Obstetrics and Gynecology, Tampere University Hospital, Tampere, Finland

${ }^{42}$ Oncogynecology, Grigorev Institute for Radiology, Kharkiv, Ukraine

${ }^{43}$ Institut Curie, Paris, Île-de-France, France

${ }^{44}$ Department of Medicine and Surgery, University of Milan-Bicocca, Milano, Italy

${ }^{45}$ Clinic of Obstetrics and Gynecology, Hospital San Gerardo, Monza, Italy

${ }^{46}$ North Estonia Medical Centre, Tallinn, Estonia

${ }^{47}$ Bellvitge University Hospital, L'Hospitalet de Llobregat, Catalunya, Spain

${ }^{48}$ Gynecology, Clinica Universitaria de Navarra, Pamplona, Navarra, Spain

${ }^{49}$ Gynecology, Clinica Universitaria de Navarra, Madrid, Spain

${ }^{50}$ Clinica Universidad de Navarra Departamento de Ginecologia y Obstetricia, Madrid, Spain

${ }^{51}$ Obstetrics and Gynecology, School of Medicine, University of Navarra, Pamplona, Spain

${ }^{52}$ Obstetrics and Gynecology, Clinica Universidad de Navarra, Madrid, Spain

Twitter Enrique Chacon @Quique_ChC, Dilyara Kaidarova @r.bolatbekova@gmail. com, Rasiah Bharathan @RasiahBharathan, Felix Boria @BoriaFelix and Juan Luis Alcazar @Juan_L_Alcazar

Collaborators On behalf of the SUCCOR study group: Nabil Abdalla, Sedat Akgöl, Demirkiran Aksahin, Shamistan Aliyev, Maria Alonso-Espias, Igor Aluloski, Claudia Andrade, Nikola Badzakov, Rosa Barrachina, Giorgio Bogani, Eduard-Aexandru Bonci, Hélène Bonsang-Kitzis, Cosima Brucker, Laura Cárdenas, Andrea Casajuana, Pere Cavalle, Jorge Cea, Benito Chiofalo, Gloria Cordeiro, Pluvio Coronado, Maria Cuadra, Javier Díez, Teresa Diniz da Costa, Santiago Domingo, Lukas Dostalek, 


\section{Original research}

Fuat Elif, Diego Erasun, Mathias Fehr, Sergi Fernandez-Gonzalez,Annamaria Ferrero, Soledad Fidalgo, Gabriel Fiol, Khadra Galaal, José García, Gerhard Gebauer, Fabio Ghezzi, Juan Gilabert, Nana Gomes, Elisabete Gonçalves, Virginia Gonzalez, Frederic Grandjean, Miriam Guijarro, Frédéric Guyon, Jolien Haesen, Gines Hernandez-Cortes, Sofía Herrero, Imre Pete, Ioannis Kalogiannidis, Erbil Karaman, Andreas Kavallaris, Lukasz Klasa, loannis Kotsopoulos, Stefan Kovachev, Uppin Arno Leht, Arantxa Lekuona, Mathieu Luyckx, Michael Mallmann, Gemma Mancebo, Aljosa Mandic, Tiermes Marina, Victor Martin, María Belén Martín-Salamanca, Víctor Lago, Alejandra Martinez, Gesine Meili, Gustavo Mendinhos, Liliana Mereu, Milena Mitrovic, Sara Morales, Enrique Moratalla, Natalia R. Gómez-Hidalgo, Bibiana Morillas, Eva Myriokefalitaki, Maja Pakižlmre, Imre Pete, Stamatios Petousis, Laurentiu Pirtea, Natalia Povolotskaya, Sonia Prader, Alfonso Quesada, Mikuláš Redecha, Fernando Roldan, Philip Rolland, Reeli Saaron, Cosmin-Paul Sarac, Jens-Peter Scharf, Špela Smrkolj, Rita Sousa, Artem Stepanyan, Vladimír Študent, Carmen Tauste, Hans Trum, Taner Turan, Manuela Undurraga, Alicia Vázquez, Ignace Vergote, George Vorgias, and Ignacio Zapardiel.

Contributors All authors contributed meaningfully to the conception or design of the work or the acquisition, analysis, or interpretation of data for the study. The authors confirm the completeness and accuracy of the data and analyses, the fidelity of the study to the protocol, and the final approval of the version to be published. LCH is responsible for the overall content as the guarantor.

Funding The authors have not declared a specific grant for this research from any funding agency in the public, commercial, or not-for-profit sectors.

Competing interests None declared.

Patient consent for publication Not applicable.

Ethics approval This study involves human participants and was approved by Beatriz González, PhD Secretary, Research Ethics Committee, April 30, 2019. The authors obtained a waiver of informed consent for this retrospective anonymized study.

Provenance and peer review Not commissioned; externally peer reviewed.

Data availability statement Data are available upon reasonable request. Data is available upon request. Please contact the corresponding author.

\section{ORCID iDs}

Enrique Chacon http://orcid.org/0000-0001-8659-8602

Nabil Manzour http://orcid.org/0000-0002-4604-6042

Denis Querleu http://orcid.org/0000-0002-3984-4812

Tayfun Toptas http://orcid.org/0000-0002-6706-6915

Mehmet Mutlu Meydanli http://orcid.org/0000-0001-6763-9720

Anna Myriam Perrone http://orcid.org/0000-0003-3140-4772

Robert Poka http://orcid.org/0000-0003-1836-1579

Marcin A Jedryka http://orcid.org/0000-0001-8935-0311

Vladyslav Sukhin http://orcid.org/0000-0002-4403-3707

Robert Fruscio http://orcid.org/0000-0001-5688-2194

Jose Angel Minguez http://orcid.org/0000-0003-3635-8920

Daniel Vázquez-Vicente http://orcid.org/0000-0002-9618-5606

Felix Boria http://orcid.org/0000-0002-4761-6190

Juan Luis Alcazar http://orcid.org/0000-0002-9700-0853

Luis Chiva http://orcid.org/0000-0002-1908-3251

\section{REFERENCES}

1 Cibula D, Pötter R, Planchamp F, et al. The European Society of Gynaecological Oncology/European Society for Radiotherapy and Oncology/European Society of Pathology guidelines for the management of patients with cervical cancer. Int J Gynecol Cancer 2018;28:641-55.
2 Ramirez PT, Frumovitz M, Pareja R, et al. Minimally invasive versus abdominal radical hysterectomy for cervical cancer. N Engl J Med 2018;379:1895-904.

3 Chiva L, Zanagnolo V, Querleu D, et al. SUCCOR study: an international European cohort observational study comparing minimally invasive surgery versus open abdominal radical hysterectomy in patients with stage IB1 cervical cancer. Int $J$ Gynecol Cancer 2020;30:1269-77.

4 Melamed A, Margul DJ, Chen L, et al. Survival after minimally invasive radical hysterectomy for early-stage cervical cancer. $N$ Engl J Med 2018;379:1905-14.

5 Doo DW, Kirkland CT, Griswold LH, et al. Comparative outcomes between robotic and abdominal radical hysterectomy for IB1 cervical cancer: results from a single high volume institution. Gynecol Oncol 2019;153:242-7.

6 National Cancer Registration and Analysis Service (NCRAS) cervical cancer surgery analysis, 2019. Available: https://www. bgcs. org. uk/ wp- content/ uploads/ 2019/ 07/ NCRAS- cervical- cancersurgeryanalysis-May- 2019- final. pdf [Accessed 03 Jul 2020].

$7 \mathrm{Kim} \mathrm{SI}$, Lee M, Lee S, et al. Impact of laparoscopic radical hysterectomy on survival outcome in patients with FIGO stage lb cervical cancer: a matching study of two institutional hospitals in Korea. Gynecol Oncol 2019;155:75-82.

8 Cusimano MC, Baxter NN, Gien LT, et al. Impact of surgical approach on oncologic outcomes in women undergoing radical hysterectomy for cervical cancer. Am J Obstet Gynecol 2019;221:619.e1-619.e24.

9 Uppal S, Gehrig P, Vetter MH, et al. Recurrence rates in cervical cancer patients treated with abdominal versus minimally invasive radical hysterectomy: a multi-institutional analysis of 700 cases. JCO 2019;37:5504-5.

10 Chen X, Zhao N, Ye P, et al. Comparison of laparoscopic and open radical hysterectomy in cervical cancer patients with tumor size $\leq 2$ cm. Int J Gynecol Cancer 2020;30:564-71.

11 Uppal S, Gehrig PA, Peng K, et al. Recurrence rates in patients with cervical cancer treated with abdominal versus minimally invasive radical hysterectomy: a multi-institutional retrospective review study. $J$ Clin Oncol 2020;38:1030-40.

12 Casarin J, Bogani G, Papadia A, et al. Preoperative conization and risk of recurrence in patients undergoing laparoscopic radical hysterectomy for early stage cervical cancer: a multicenter study. $J$ Minim Invasive Gynecol 2021;28:117-23.

13 Manzour N, Chacon E, Martín-Calvo N. 258 Design and validation of a recurrence risk predicting score in early stage cervical cancer after radical hysterectomy. Int J Gynecol Cancer 2020;30.

14 Pecorelli S, Zigliani L, Odicino F. Revised FIGO staging for carcinoma of the cervix. Int J Gynaecol Obstet 2009;105:107-8.

15 Boria F, Chiva L, Zanagnolo V, et al. Radical hysterectomy in early cervical cancer in Europe: characteristics, outcomes and evaluation of ESGO quality indicators. Int J Gynecol Cancer 2021;31:1212-9.

16 Bizzarri N, Pedone Anchora L, Kucukmetin A, et al. Protective role of conization before radical hysterectomy in early-stage cervical cancer: a propensity-score matching study. Ann Surg Oncol 2021;28:3585-94.

17 Schmeler KM, Pareja R, Lopez Blanco A, et al. ConCerv: a prospective trial of conservative surgery for low-risk early-stage cervical cancer. Int J Gynecol Cancer 2021;31:1317-25.

18 Hutchcraft ML, Smith B, McLaughlin EM, et al. Conization pathologic features as a predictor of intermediate and high risk features on radical hysterectomy specimens in early stage cervical cancer. Gynecol Oncol 2019;153:255-8.

19 Martín-Calvo N, Chacón E. Can a cone biopsy predict nodal status in early cervical cancer? 22nd European Congress on Gynecological Oncology, European Society of Gynaecological Oncology, 2020.

20 Köhler C, Hertel H, Herrmann J, et al. Laparoscopic radical hysterectomy with transvaginal closure of vaginal cuff - a multicenter analysis. Int J Gynecol Cancer 2019;29:845-50. 